\title{
Long Noncoding RNA X-Inactive Specific Transcript Facilitates Cellular Functions in Melanoma via miR-I39-5p/ROCKI Pathway
}

This article was published in the following Dove Press journal: OncoTargets and Therapy

\author{
Ke Tian',* \\ Dongxia Sun ${ }^{2, *}$ \\ Min Chen ${ }^{2}$ \\ Yifei Yang' \\ Fang Wang ${ }^{3}$ \\ Taotao Guo ${ }^{4}$ \\ Zhimin Shi $\mathbb{D}^{3}$ \\ 'Department of Dermatology, Affiliated \\ Hospital of Hebei University of \\ Engineering, Handan 056000, Hebei \\ Province, People's Republic of China; \\ ${ }^{2}$ Department of Pathology, Handan \\ Maternal and Child Health Hospital, \\ Handan 056000, Hebei Province, People's \\ Republic of China; ${ }^{3}$ Department of \\ Pathology, Medical College, Hebei \\ University of Engineering, Handan \\ 056000, Hebei Province, People's \\ Republic of China; ${ }^{4}$ Laboratory \\ Department, Handan Second Hospital, \\ Handan, Hebei Province 056000, People's \\ Republic of China
}

*These authors contributed equally to this work
Purpose: Although X-inactive specific transcript (XIST) is known to play a critical role in the pathogenesis of melanoma, the mechanisms through which this remains unclear.

Methods: RNAseq, immunohistochemistry, and qRT-PCR were used to identify the levels of XIST, miR-139-5p, and Rho-Associated Coiled-Coil Containing Protein Kinase-1 (ROCK1) in melanoma tissues and cells. A subcellular fractionation assay was used to determine the location of XIST. CCK- 8 and colony formation assays were used to evaluate cellular proliferation. Cell migration and wound healing assays were used to detect the effects on cell migration. RNA pull-down was used to confirm the interaction between XIST and miR-139-5p. Besides, the xenograft tumor experiment was performed to further verify the roles of XIST in melanoma.

Results: In this study, an increased level of XIST was revealed in melanoma tissues and cells, which was associated with higher TNM stage and positive lymph node metastasis. XIST was found to function as a "molecular sponge" of miR-139-5p to facilitate cellular functions. Moreover, these consequences could be partially reversed by inhibition of miR139-5p. MiR-139-5p was found to target ROCK1 directly, leading to suppression of ROCK1 expression; this effect could be partially reversed by inhibiting XIST expression. Furthermore, the deletion of ROCK1 induced anti-oncogenic effects similar to those seen with knockout of XIST. Upregulation of miR-139-5p and knockdown of XIST could inhibit cell functions in melanoma.

Conclusion: Our findings suggested that the lncRNA XIST facilitates cellular functions in melanoma via the miR-139-5p/ROCK1 pathway.

Keywords: LncRNA, XIST, melanoma, MiR-139-5p, ROCK1

\section{Introduction}

Melanoma is one of the most prevalent malignancies with an estimated 200,000 new cases and 46,000 occurring globally each year. ${ }^{1}$ Previous findings have demonstrated favorable prognoses for melanoma patients at early stages (I and II), but poor prognoses for melanoma patients at advanced stages (III and IV). ${ }^{2}$ Due to melanoma's susceptibility to metastasis, the prognosis of melanoma patients with advanced disease is unfavorable, with five-year survival rates below $20 \%{ }^{3}$ Therefore, clarifying the mechanisms of melanoma pathogenesis and establishing biomarkers for early diagnosis and therapy would be a significant milestone in improving patient outcomes.

Long non-coding RNAs (lncRNAs) are a sub-class of non-coding RNAs (ncRNAs) with abundant and diverse functions. ${ }^{4}$ Despite limited protein-coding potential,
Correspondence: Zhimin Shi

Tel +86-17732027089

Email hdkosoier283@163.com 
lncRNAs can exert important regulatory functions on both the transcriptional and post-transcriptional levels. ${ }^{5}$ Previous studies have shown the $\mathrm{X}$ inactive-specific transcript (XIST) to be dysregulated in various malignancies and associated with malignancy progression. ${ }^{6-8}$ XIST has been reported to act both as an oncogene and as an anti-oncogene across various types of malignancies. XIST was found to act as a cancer-promoting gene in bladder cancer, and downregulation of XIST was shown to inhibit cell proliferative and migratory capacity via p53 and Tet Methylcytosine Dioxygenase 1 (TET1). ${ }^{9}$ Upregulation of XIST has been found in retinoblastoma tissues and cells. Upon reduction of XIST levels, retinoblastoma cell proliferation is inhibited and cell apoptosis increased, demonstrating XIST to act as an oncogene in retinoblastoma. ${ }^{10}$ In cervical cancer, XIST was found to be elevated in tumor tissues and cells and further defined as an oncogene promoting cancer progression via the miR-200a/Fus pathway. ${ }^{11}$ In breast cancer, downregulation of XIST was found in tumor tissues and cells, and cell proliferative, migratory and invasive capacity was inhibited after upregulation of XIST, showing XIST to act as an antioncogene in this case. ${ }^{12}$ In prostate cancer, downregulation of XIST has been found in tumor specimens and cells. Cellular proliferation and metastasis were inhibited by XIST, demonstrating that XIST acts as an anti-oncogene in prostate cancer. ${ }^{13}$ However, the crucial functions of XIST in the pathogenesis of melanoma have not previously been identified.

Therefore, we assessed XIST levels in melanoma tissues and cells and further investigated the role of XIST on cell behaviors, including cell proliferation and invasion. Finally, we explored the underlying mechanism and pathway through which XIST exerts its impact on melanoma cell proliferation and invasion.

\section{Materials and Methods}

\section{Patients and Melanoma Specimens}

Melanoma specimens and para-carcinoma specimens were recruited from 62 patients who had tumors removed at Affiliated Hospital of Hebei University of Engineering, Handan Maternal and Child Health Hospital and Handan Second hospital between 2015 and 2018. None of the patients received therapy or had other tumors prior to surgery. Corresponding para-carcinoma tissues were obtained more than $3 \mathrm{~cm}$ from the tumor margin. Histopathological diagnosis was performed under the management of two experienced pathologists. After the fresh tissues were collected, they were flash-frozen in liquid nitrogen and stored at $-80^{\circ} \mathrm{C}$. The current research was managed by the Ethics Committees of the three aforementioned hospitals. All patients read and signed informed consent agreements prior to this experiment.

\section{Immunohistochemistry}

Three-millimeter tumor sections were incubated with commercial rabbit polyclonal antibodies against XIST (Santa Cruz Biotechnology) at $1 / 100$ dilution overnight at $4{ }^{\circ} \mathrm{C}$. Then, the sections were conjugated with horseradish peroxidase (HRP) antibody (1:500 dilution; Santa Cruz Biotechnology, Santa Cruz, CA) at room temperature for 2 hrs, then covered by 3, 3-diaminobenzidine (DAB) (Vector Laboratories, Burlingame, CA), and slides were mounted with Vectashield mounting medium (Vector Laboratories). Subsequently, all fields were observed under light microscopy (Olympus 600 auto-biochemical analyzer, Tokyo, Japan). Control experiments without primary antibody demonstrated that the signals observed were specific.

\section{Differential Analysis of IncRNA Based on RNA-Seq}

In total, 20 human melanoma specimens and para-carcinoma specimens from 10 patients with melanoma were processed for whole transcriptomic analysis by RNA-seq. Long ( $>200$ bp) transcripts without any ORFs or protein domain that failed to encode any proteins were considered as novel lncRNA candidates. DEGseq software (v1.14.0) was used to identify differentially expressed lncRNA candidates, and lncRNAs with $\mid \log _{2}$ (fold change) $\mid>2$ and false discovery rate. $^{14}$ (FDR) $<0.05$ were identified as significant lncRNA regulators in melanoma.

\section{Cell Culture and Transfection}

Melanoma cell lines (A375 (ATCC ${ }^{\circledR}$ CRL-1619), SK-MEL -110 (ATCC ${ }^{\circledR}$ HTB-67), HS-1 (ATCC ${ }^{\circledR}$ CRL-9446), MEL-

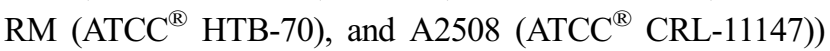
and normal human epidermal melanocytes (HEMa-LP ATCC $^{\circledR}$ PCS-200-013)) were obtained from American Type Culture Collection and cultivated in Dulbecco's Modified Eagle Medium (DMEM, Gibco-BRL, Grand Island, NY, USA) and Medium 254 (Cascade Biologics, Portland, OR, USA) containing 10\% fetal bovine serum (FBS; SigmaAldrich, St. Louis, MO), penicillin $(100 \mu \mathrm{L} / \mathrm{mL})$, streptomycin $(100 \mathrm{mg} / \mathrm{mL})$ and glutamine. All cells were maintained in 
a $37^{\circ} \mathrm{C}$ atmosphere that containing $5 \% \mathrm{CO}_{2}$. pcDNA-XIST (Rho-associated coiled-coil containing protein kinase 1, XISToverexpressing plasmid), si-XIST (siRNA targeting XIST), siROCK1 (siRNA targeting ROCK1), si-NC (negative control siRNAs), miR-139-5p mimic (overexpressing oligonucleotides) and mimic NC (negative control) were all obtained from GenePharma (Shanghai, China). Lipofectamine 3000 (Invitrogen) was utilized for transfecting all oligonucleotides and plasmids into melanoma cells.

\section{Quantitative Real-Time Polymerase Chain Reaction (qRT-PCR)}

RNA extraction from melanoma tissues and cells was carried out with TRIzol Reagent (Invitrogen). cDNA synthesis was conducted using the extracted RNA and Reverse Transcription Kit (Qiagen $\mathrm{GmbH}$ ). After that, qRT-PCR was using SYBR-Green PCR Master Mix kits (Qiagen $\mathrm{GmbH}$ ) and the Roche LightCycler 480 RT-qPCR System. Relative gene expression between groups was determined by $2^{-\Delta \Delta \mathrm{Ct}}$ method normalized to GAPDH or U6 expression. Primer sequences are shown in Table S1.

\section{Cell Migration Assay}

The wound healing assay was carried out using a digital camera system (Olympus Corporation, Tokyo, Japan). Once HS-1 cells reached 95\% convergence, a disposable pipette tip was used to create a straight wound in the culture. After being washed with PBS, the remaining cells were incubated for $24 \mathrm{hrs}$. Images were taken at 0 and 24 hrs so that wound closure could be assessed.

\section{Cell Counting Kit-8 (CCK-8) Assay}

Proliferative ability of melanoma cells was determined using the CCK-8 assay (Everbright lnc). In short, after transfection, HS-1 cells were planked in 96-well plated in 96-well plates $\left(3 \times 10^{3}\right.$ cells/well $)$ and incubated for $8 \mathrm{hrs}$. Following this, $10 \mu \mathrm{L}$ CCK-8 solution was added to each well and cells were incubated for an additional 90 mins. Optical density at $450 \mathrm{~nm}$ was measured every $24 \mathrm{hrs}$ (for a total of $72 \mathrm{hrs}$ ) with an ELISA microplate reader (Thermo Labsystems, Waltham, MA, USA).

\section{Cell Invasion Assay}

A transwell chamber (BD Biosciences, New York, NJ, USA) pre-coated with Matrigel was utilized to assess cells' invasive capability. In short, the same amount of transfected melanoma cells in $100 \mu \mathrm{L}$ serum-free medium were placed in the top chamber of each transwell, while $450 \mu \mathrm{L}$ DMEM medium and $50 \mu \mathrm{L}$ FBS was placed in the bottom chamber as an attractant. After 36 hrs of cultivation, the cells in the top chamber were washed and cleaned while cells across the membrane were fixed with paraformaldehyde and stained with crystal violet and cell numbers were counted under a light microscope.

\section{Subcellular Fractionation Assay}

RNA isolated from nuclear or cytoplasm fraction via the Nuclear/Cytosol Fractionation Kit (Biovision, San Francisco Bay, CA, USA) was evaluated with qRT-PCR analysis. U6 or GAPDH acted as the nuclear or cytoplasm fractions by Nuclear and Cytoplasmic Extraction Reagents (Thermo Fisher Scientific).

\section{Luciferase Reporter Assays}

PCR was utilized to amplify related sequences of XIST and ROCK1-3'UTR including predicted miR-139-5p binding sequences. Sequences were cloned into a pmiRGLO reporter vector (Promega, USA) in order to generate wild type XIST reporter (XIST-wt) or ROCK1 reporter (ROCK1-wt) constructs. Mutant XIST reporter (XISTmut) and ROCK1 reporter (ROCK1-mut), whose binding sites were mutated, were produced using the rapid sitespecific mutation kit (Tiangen Biotech, Beijing, China). Lipofectamine 3000 (Invitrogen) was utilized to cotransfect reporter constructs and miR-139-5p mimic or $\mathrm{NC}$ into melanoma cells. After $36 \mathrm{hrs,}$, relative luciferase activities were measured using a dual-luciferase reporter assay system (Promega Corporation, USA). Changes in ROCK1 levels were determined after normalization to GAPDH.

\section{Western Blot Analysis}

RIPA buffer (Sigma-Aldrich, St Louis, MO, USA) was utilized for cell lysis. Proteins were separated using 10\% SDSPAGE gel and then transferred to polyvinylidene difluoride (PVDF) membranes. Then, the membranes were blocked with 5\% BSA in Tris-buffered saline with $0.1 \%$ Tween 20 (TBST), followed by incubation with primary antibodies against ROCK1 (Abcam, Cambridge, MA, USA) and GAPDH (Santa Cruz Biotechnology, Santa Cruz, CA, USA) at $4^{\circ} \mathrm{C}$ overnight. After that, the membranes were incubated with horseradish peroxidase-conjugated secondary antibody, and the blots were measured and visualized using a Bio-Rad Gel Doc XR + system (Bio-Rad, Hercules, CA, USA). 


\section{RNA Immunoprecipitation (RIP) Assay}

EZ-Magna RIP RNA-binding protein immunoprecipitation kit (Millipore, Bedford, MA, USA) was used to perform the RIP assay. Cell lysates were incubated in RIP buffer containing magnetic beads conjugated to argonaute-2 (Ago2) (Millipore) antibody or negative control IgG (Millipore). qRT-PCR was carried out on the purified immunoprecipitated RNA to measure relative levels of XIST and miR-139-5p.

\section{RNA Pull-Down Assay}

This assay was performed as previously described by Rivetti et al ${ }^{15}$ Melanoma cells were transfected with either biotinylated XIST or, as a negative control, a biotinylated IncRNA without complementary to miR-139-5p. Cell lysates were then incubated for $48 \mathrm{hrs}$ with M-280 streptavidin magnetic beads (Invitrogen). qRT-PCR was performed to measure miR-193-5p levels.

\section{Bioinformatics Analysis}

StarBase version 3.0 (http://starbase.sysu.edu.cn/index.php) was utilized to predict the target of XIST. TargetScan (http:// www.targetscan.org) and starBase version 3.0 were utilized to predict downstream genes regulated by miR-139-5p. LinkedOmics (http://www.linkedomics.org/) is a publicly available portal that includes multi-omics data from all 32 TCGA Cancer types. ${ }^{16}$ The LinkFinder module was used to verify the correlations between miR-139-5p level and ROCK1 level, as well as between XIST level and ROCK1 level.

\section{Tumor Xenograft Model in vivo}

All animal experiments were approved by the Animal Care and Use Committee of Handan Maternal and Child Health Hospital and Handan Second hospital. A total of 10 female nude mice (BALB/c nude mice, Vitalriver, Nanjing, China; 6 weeks old) were randomly divided into 2 groups: si-XIST and si-NC. Stably transfected cells were inoculated subcutaneously into the flank of nude mice. Tumors were measured with vernier calipers every 4 days, and the mice were euthanized after 3 weeks. The volume of the implanted tumor was calculated by using the formula: volume $=\left(\right.$ width $^{2} \times$ length $) / 2$. Gene expression in the implanted tumor was detected by qRT-PCR analysis.

\section{Statistical Analysis}

$\mathrm{P}<0.05$ was applied as the threshold for statistical significance. Graphpad Prism 7 software was used for data analysis. Variance between two or more groups was assessed via Student's $t$-test and one-way ANOVA. Significant differences in gene expression between melanoma specimens and corresponding normal specimens were evaluated using the Wilcoxon matched-pairs test. Correlations among XIST, miR-139-5p, and ROCK1 were evaluated using Pearson correlation analysis.

\section{Results}

\section{XIST Is Upregulated in Melanoma Tissues} and Cell Lines

After comparing the mean TPM value of the melanoma group with that of the para-carcinoma group, three transcripts were selected from 857 lncRNAs according to the criteria: $\mid \log 2$ (fold change) $\mid>2, \quad \mathrm{FDR}<0.05$ (one upregulated and two downregulated). A heat map of this data is displayed in Figure 1A, showing that the upregulated lncRNA was XIST. Therefore, we employed qRT-PCR to measure XIST level in tissues and cells. Figure 1B shows that a significantly higher level of XIST was observed in melanoma specimens when compared to corresponding normal skin specimens $(\mathrm{P}<0.001)$. Furthermore, downregulation of XIST was dramatically associated with negative lymph node metastasis $(\mathrm{P}<0.001)$, matutinal TNM stage $(\mathrm{P}<0.025)$, as shown in Table $\mathrm{S} 2$. The result of immunohistochemistry showed high XIST protein expression in melanoma tissues in comparison with normal tissues (Figure 1C). Similar upregulation of XIST was also observed in melanoma cell lines. A significantly enhanced level of XIST was found in melanoma cells (Figure 1D, all $\mathrm{P}<0.001)$ compared to HEMa-LP cells. All of these findings support the idea that aberrant XIST levels play a role in melanoma progression.

\section{XIST Functions as a Molecular Sponge of miR-139-5p in Melanoma Cells}

A growing body of research suggests that lncRNAs may function as "sponges" of miRNAs, such that these lncRNAs competitively absorb miRNAs and prevent their interactions with target mRNAs. Subcellular fragmentation assay is performed, which revealed that XIST is located in the cytoplasm (Figure 2A). In our study, starBase version 3.0 (http://starbase.sysu.edu.cn) was utilized to predict potential target miRNAs of XIST. According to the data, miR-139-5p was selected as potential miRNA of interest. Figure $2 \mathrm{~B}$ shows the potential binding sites. The amount of miR-139-5p was detected, 

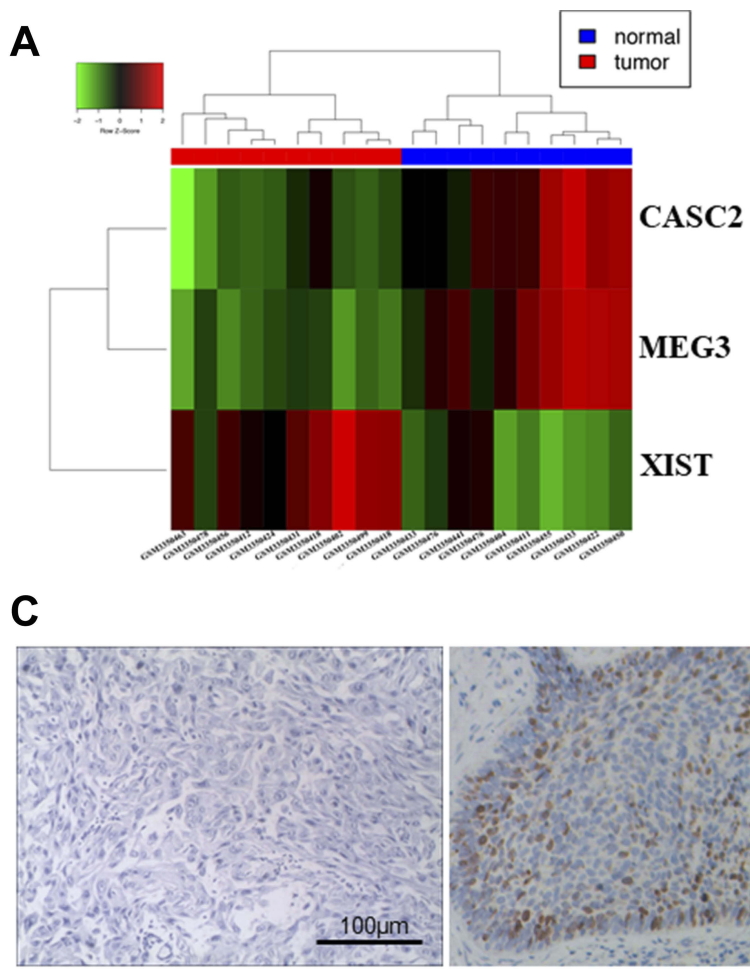

Normal tissues

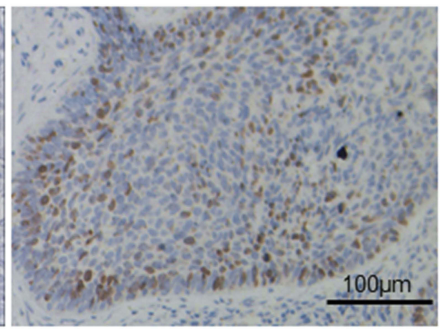

Melanoma tissues
B

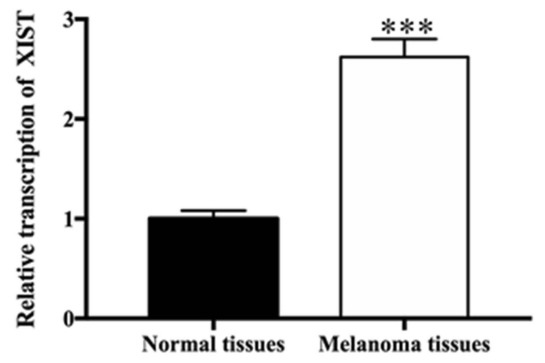

D

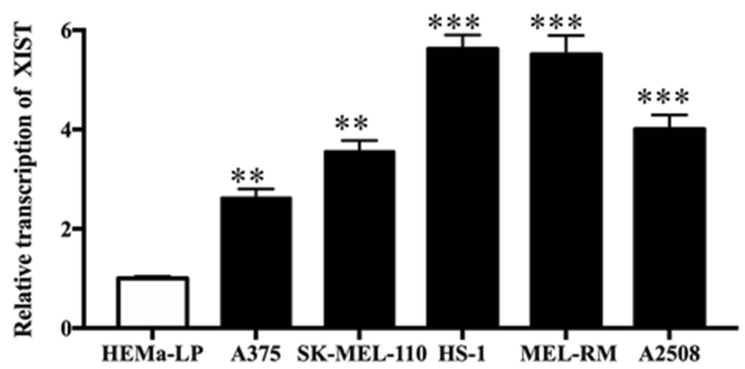

Figure I XIST level in melanoma tissues and cell lines.

Notes: (A) Representative microarray analysis of XIST in melanoma tissue tissues and normal tissues (red denotes high relative expression and blue denotes low relative expression). (B) Relative level of XIST in melanoma tissues and normal tissues. (C) Immunohistochemistry showing general tissue morphology of melanoma tissues and normal tissues (Bar is $100 \mu \mathrm{m}$ ). (D) Relative levels of XIST in melanoma cell lines. $* * \mathrm{P}<0.0 \mathrm{I}, * * * \mathrm{P}<0.00 \mathrm{I}$.

as well as the correlation between miR-139-5p level and XIST level. Figure 2C shows the level of miR-139-5p in melanoma samples and reveals decreased miR-139-5p expression in melanoma tissues as compared with normal tissues. As shown in Figure 2D, the luciferase activity of the XIST-wt reporter was remarkably repressed after upregulation of miR-139-5p $(\mathrm{P}<0.05)$ while that of XIST-mut reporter was not affected. Moreover, XIST level was negatively associated with miR-139-5p level in melanoma tissues (Figure 2E, $\mathrm{P}<0.001, \mathrm{R}^{2}=0.6751$ ). We then performed verification assays to investigate the direct relationship between XIST and miR-139-5p. Data from RIP assays demonstrate that XIST level and miR-139-5p level were both significantly enriched when incubated with Ago2 antibody in comparison to control IgG (Figure 2F). Moreover, data from RNA pull-down assays revealed a significant enrichment of miR-139-5p in the metastasisassociated lung adenocarcinoma transcript 1 pulled down pellets as compared to a matched group (Figure 2G, $\mathrm{P}<0.01)$. We then explored the relationship between XIST level and miR-139-5p level in melanoma cells by transfecting melanoma cells with si-XIST. As anticipated, XIST level declined (Figure $2 \mathrm{H}, \mathrm{P}<0.05$ ) while miR-139$5 \mathrm{p}$ level increased (Figure 2I, $\mathrm{P}<0.01$ ) after XIST knockdown.

\section{Knockdown of XIST Suppresses Melanoma Cellular Functions by Regulating miR-I39-5p}

To assess the cellular functions and potential mechanism of XIST, melanoma cells were transfected with specifically designed oligonucleotides or/and plasmids. As shown in Figure $3 \mathrm{~A}$, knockdown of XIST hindered the proliferation of melanoma cell $(\mathrm{P}<0.01)$, while this effect was partially reversed upon transfection with miR-139-5p inhibitor $(\mathrm{P}<0.01)$. As shown in Figure $3 \mathrm{~B}$ and $\mathrm{C}$, migration ability declined in si-XIST-transfected cells. These effects were also partially reversed upon transfection with miR-139-5p inhibitor $(\mathrm{P}<0.01)$. Moreover, the transwell assay revealed a declined invasion ability in si-XIST-transfected cells, which was again partially reversed after transfection with miR-139-5p inhibitor (Figure 3D and E). Altogether, 


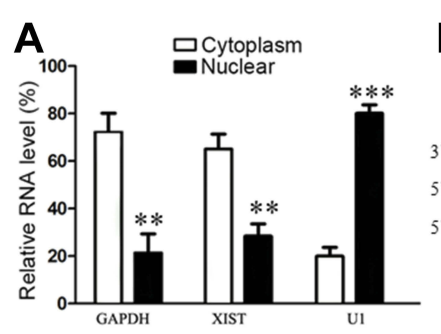

B

3'-tgaccttgtgcaCGTGACATCt-5' miR-139-5p

5'-agccettctcttaGAACTGTAGa--3' XIST-WT

5'-agagccettctettaAAGTCACGAa-3' XIST-MUT
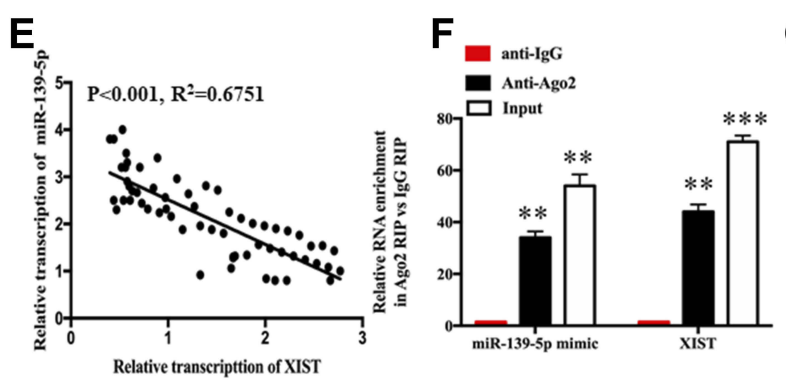
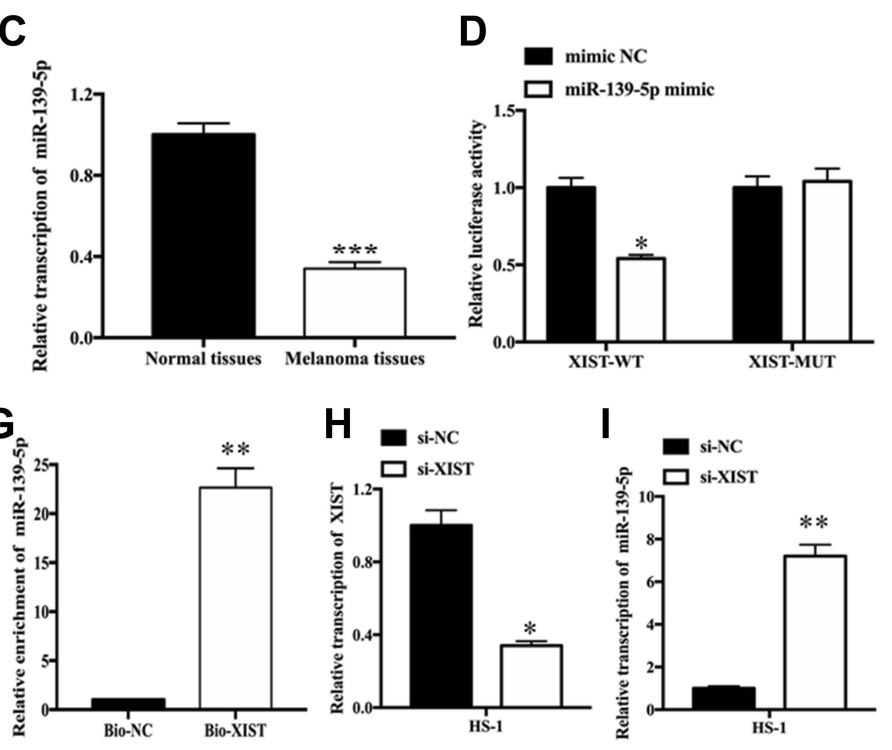

Figure 2 XIST inhibits miR-139-5p expression by acting as a molecular sponge in melanoma cells.

Notes: (A) Relative RNA level of XIST in cytoplasm and nuclear. (B) Binding sequences between XIST and miR-I39-5p. (C) Relative levels of miR-I39-5p in melanoma tissues and normal tissues. (D) Negative correlation between XIST expression and miR-I39-5p expression in melanoma tissues. (E) Luciferase activity in HS-I cells cotransfected with XIST-wt or XIST-mut reporter and miR-I39-5p mimic or miR-NC. (F) RIP assays were performed using ago2 antibody or control IgG antibody in HS-I cells. (G) RNA pull-down assay was conducted to assess binding between XIST and miR-I39-5p in HS-I cells. (H, I) qRT-PCR analysis of XIST and miR-I39-5p expressions in HS-I cells after transfection with si-NC or si-XIST. Bio-NC, a biotinylated IncRNA that is not complementary to miR-I39-5p was employed as a negative control. $* \mathrm{P}<0.05$, $* * \mathrm{P}<0.01$, *** $\mathrm{P}<0.001$.

Abbreviations: mut, mutant; qRT-PCR, quantitative reverse transcription-polymerase chain reaction; RIP, RNA immunoprecipitation; si-NC, scrambled RNA for negative control; si-XIST, small nucleolar RNA XIST; WT, wild type; MUT, mutant type.

knockdown of XIST had an anti-oncogene function in melanoma through regulation of miR-139-5p in melanoma.

We also explore the role of miR-139-5p in melanoma. As expected, upregulation of miR-139-5p could inhibit cell proliferation, migration, and invasion. As shown in Figure 3F, upregulation hindered the proliferation of melanoma cell $(\mathrm{P}<0.05)$. Besides, the transwell assay revealed a declined invasion ability in miR-139-5p mimic-transfected cells compared with that in mimic NC-transfected cells (Figure 3G and $\mathrm{H}, \mathrm{P}<0.001)$. Moreover, migration ability declined in miR139-5p mimic-transfected cells (Figure 3I and J, $\mathrm{P}<0.05$ ).

\section{ROCKI Mediates the Effects of XIST/}

\section{miR-I39-5p on Melanoma Progression}

TargetScan (http://www.targetscan.org) and starBase version 3.0 were utilized to predict downstream genes regulated by miR-139-5p. ROCK1 was selected as a potential downstream target gene, and the binding sequences of miR-139-5p are shown in Figure 4A. Elevation of miR-139-5p levels led to subdued luciferase activity of the ROCK1-wt reporter in HS-1 cells; these effects could be partially reversed by upregulating XIST levels (Figure 4B). However, no significant changes were found in the ROCK1-mut reporter luciferase activity (Figure 4B). Western blot was conducted to assess the impact of XIST or miR-139-5p levels on ROCK1 protein levels in melanoma cells. Increased levels of miR-139-5p and decreased levels of XIST both led to a significant decline in ROCK1 protein levels, and these effects could be partly reversed via transfection of miR-139-5p inhibitor (Figure 4C and D). Interestingly, a negative relationship was found between miR-139-5p levels and ROCK1 levels (Figure 4E, $\mathrm{P}<0.001, \mathrm{R}^{2}=0.597$ ), while a positive correlation was found between XIST and ROCK1 levels (Figure 4F, $\mathrm{P}<0.001$, $\left.\mathrm{R}^{2}=0.4059\right)$. Publically available data of TCGA also revealed a positive correlation between XIST level and ROCK1 level (Figure $\mathrm{S} 1 \mathrm{~A}, \mathrm{P}=0.002, \mathrm{R}^{2}=0.088$ ), and a negative relationship between miR-139-5p and ROCK1 levels (Figure S1B, $\left.\mathrm{P}=0.033, \mathrm{R}^{2}=0.047\right)$. As a whole, these data suggest that XIST modulates ROCK1 level via "sponging" miR-139-5p.

\section{Knockdown of ROCKI Suppresses Cell} Functions in Melanoma

We also explore the role of ROCK1 in melanoma. As expected, knockdown of XIST could hinder cell proliferation, migration, and invasion. As shown in Figure 5A, knockdown of XIST hindered the proliferation of melanoma cell $(\mathrm{P}<0.05)$. Besides, the transwell assay revealed a declined invasion ability in si-XIST-transfected cells 

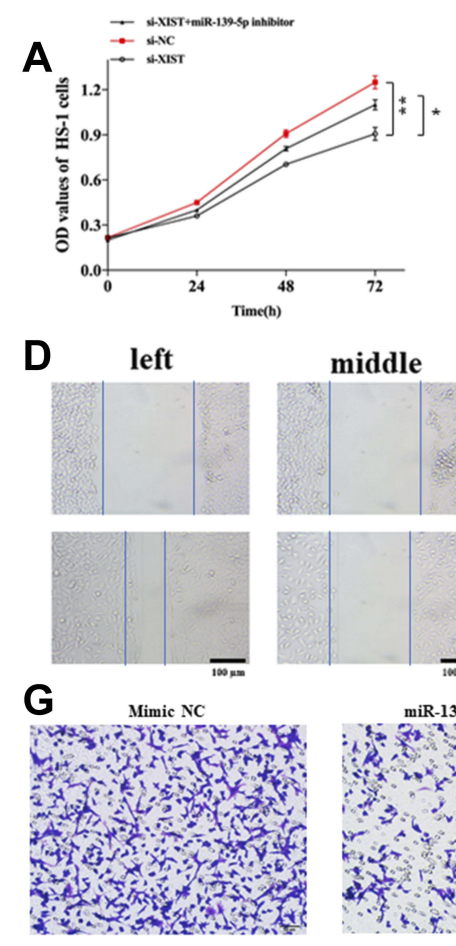

middle
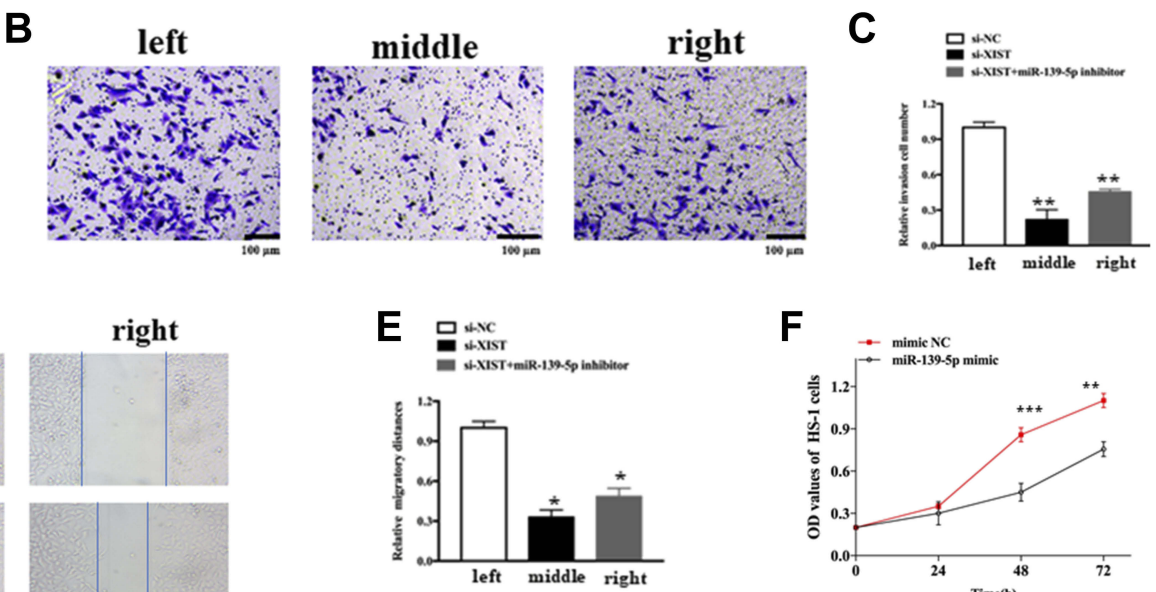

F
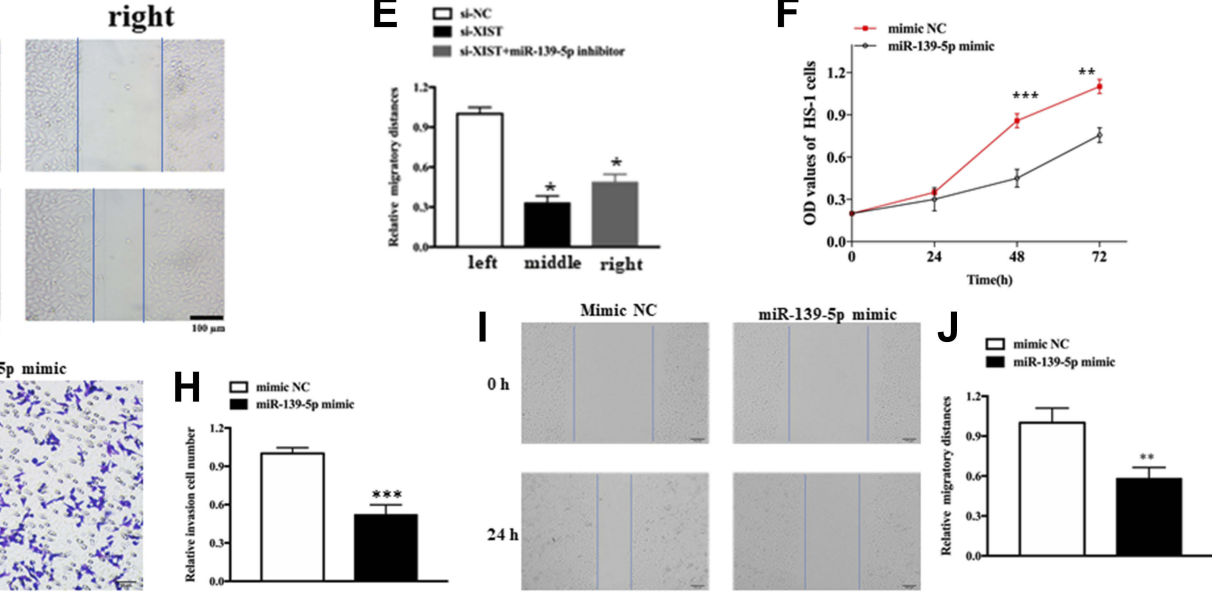

Figure 3 The role of XIST in cellular function.

Notes: (A) Cell proliferation in HS-I cells after transfection with either XISTalone or together with miR-I39-5p inhibitor. (B and C) Cell migration in HS-I cells after transfection with either XIST alone or together with miR-139-5p inhibitor. (D and E) Cell invasion in HS-I cells after transfection with either XIST alone or together with miR-I39-5p inhibitor. (F) Cell proliferation in HS-I cells after transfection with miR-I39-5p mimic or mimic NC (G and $\mathbf{H})$ Cell invasion in HS-I cells after transfection with either miR-I39-5p mimic or mimic NC. (I and J) Cell migration in HS-I cells after transfection with miR-I39-5p mimic or mimic NC. All bars are I00 $\mu \mathrm{m}$. $* \mathrm{P}<0.05$, $* * \mathrm{P}<0.0 \mathrm{I}, * * * \mathrm{P}<0.00 \mathrm{I}$.

Abbreviations: OD, optical density; si-NC, scrambled RNA for negative control.

compared with that in si-NC-transfected cells (Figure 5B, $\mathrm{P}<0.001)$. Moreover, migration ability declined in si-XISTtransfected cells (Figure 5C, $\mathrm{P}<0.01$ ).

\section{XIST Promotes Tumor Growth of TNBC Cells in vivo by Regulating miR-139-5p/ ROCKI Pathway}

In order to investigate the effects of XIST expression on tumor growth in vivo, HS-1 cells transfected with si-XIST or si-NC were injected subcutaneously into nude mice xenograft model, respectively. Compared with the negative control, tumor weight showed a significant decrease in siXIST group. Consistent with in vitro cell growth results, tumor growth rate was remarkably lower in the si-XIST group than in the negative control group (Figure 6A and B). In the implanted tumor, miR-139-5p level was significantly higher and ROCK1 level was significantly lower in si-XIST group (Figure 6C-E).

\section{Discussion}

Previous studies have identified associations between lncRNAs and melanoma pathogenesis, with these effects mediated via modulation of molecular pathways and interactions. In our research, we aimed to exhaustively probe the functions and potential mechanisms of XIST in melanoma.

In our study, we first explored the level of XIST in melanoma tumor tissues as well as cell lines, revealing XIST to be upregulated in both melanoma tissues and cell lines. Our results are consistent with those of a previous study. ${ }^{17}$ We next explored the function of XIST in melanoma and found that knockdown of XIST suppressed melanoma cellular functions (proliferation, migration, and invasion). Another study also suggested that XIST could promote melanoma progression, ${ }^{17}$ demonstrating XIST may act as an oncogene in melanoma. Moreover, XIST has been shown to act as an oncogene in other types of cancer. Chen et al revealed that up-regulated XIST acts as an oncogene in cervical cancer and contributes to progression of tumor cells. ${ }^{18}$ In gastric cancer, XIST is suggested to accelerate cell growth and invasion via the miR-497/ MACC1 axis. ${ }^{19}$

Previous studies have indicated that lncRNAs may function as miRNA sponges and interact with both miRNAs and mRNAs. ${ }^{20}$ MiR-139-5p was discovered to be connected to 
A 3'-ugaccucugugcacgUGACAUCu-5' miR-139-5p

5'-aacuucagugcccucACUGUAGa-3' ROCK1-WT

5'-aaacuucagugcccucUGACAUCAa-3' ROCK1-MUT

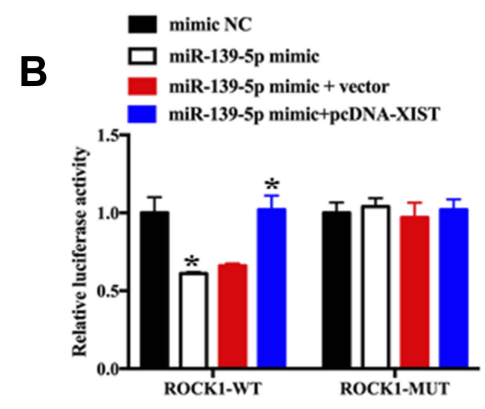

E

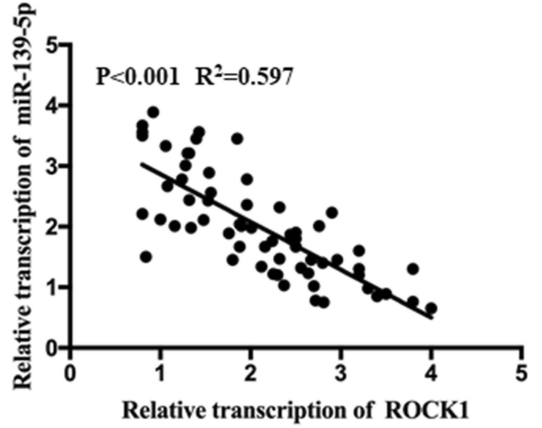

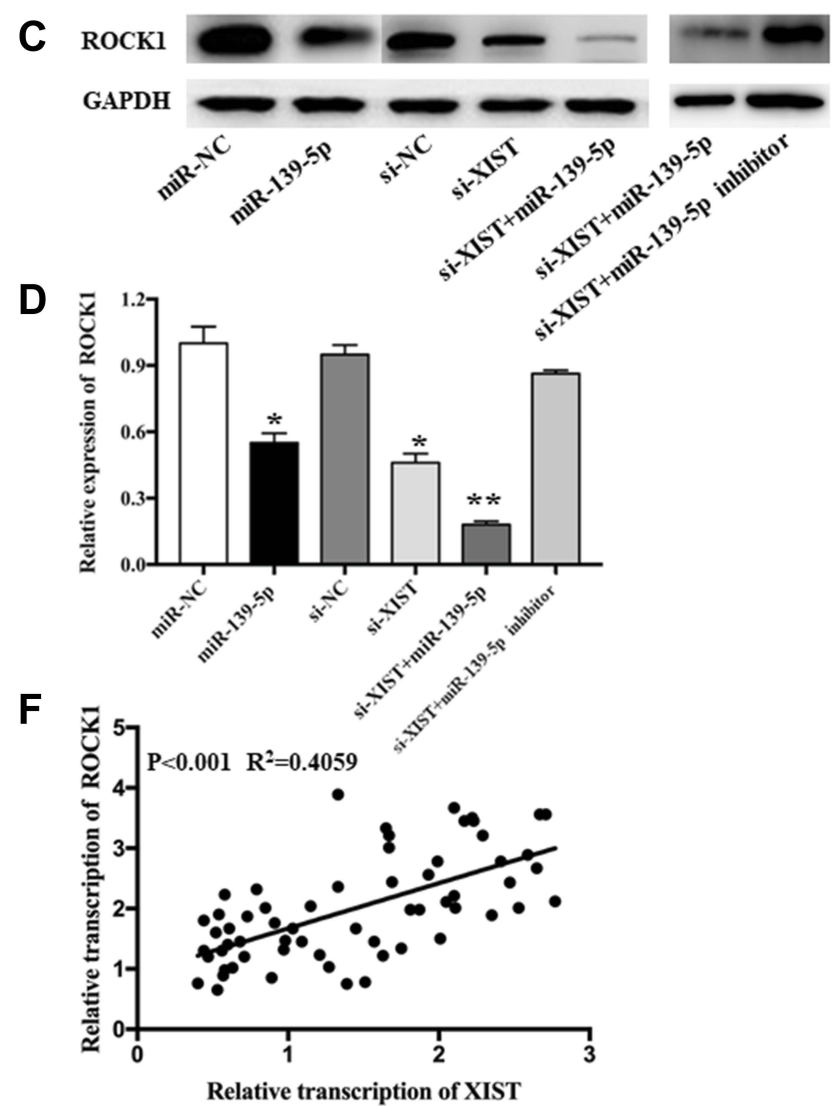

Figure 4 The anti-oncogene roles of ROCKI silencing are mediated by XIST/miR-I39-5p.

Notes: (A) Binding sequence between miR-I39-5p and ROCKI-3'UTR region. (B) Luciferase activity was detected in HS-I cells co-transfected with ROCKI-wt or ROCKI-mut reporter and miR-I39-5p mimic, mimic NC, miR-I39-5p+vector, or miR-139-5p+pcDNA-XIST. (C and D) Protein level of ROCKI in HS-I cells with miR-I39$5 p$ overexpression, XIST knockdown, or miR-139-5p inhibitor. All experiments were performed in triplicate and repeated three times. (E and $\mathbf{F})$ Correlation assay between ROCKI and miR-139-5p or XIST in melanoma tumor tissues. ${ }^{*} \mathrm{P}<0.05$, $* * \mathrm{P}<0.01$.

Abbreviations: WT, wild type; MUT, mutant type; miR-NC, scrambled RNA for negative control.

tumor cell functions in various cancers. In prostate cancer, decreased levels of miR-139-5p were found in cancer specimens, and elevated amounts of miR-139-5p were shown to restrain tumor cellular functions (proliferation and migration) by regulating SRY-box transcription factor 5 (SOX5). ${ }^{21}$ Moreover, in osteosarcoma tissues, significantly decreased levels of miR-139-5 $p$ were found, and miR-139$5 \mathrm{p}$ was shown to inhibit tumor cell growth and invasion. ${ }^{22}$ In bladder cancer, decreased amounts of XIST suppressed cell proliferation and invasion by regulating the $\mathrm{Wnt} / \beta$ catenin pathway. However, these effects were diminished after loss of miR-139-5p. ${ }^{23}$ In our study, a potential miRNA and mRNA regulated by XIST in melanoma were explored. StarBase 3.0 was utilized to identify miR-139-5p as one of the targets of XIST. Significantly decreased levels of miR$139-5 p$ were found in melanoma, and a negative correlation between XIST and miR-139-5p levels in melanoma was also identified. Further verification experiments (luciferase reporter, RIP, and RNA pull-down assay) revealed a direct binding between XIST and miR-139-5p. Furthermore, when XIST levels were decreased in HS-1 cells, miR-139$5 p$ levels became elevated. In brief, XIST could suppress miR-139-5p by direct interaction. We then probed the roles of miR-139-5p on XIST in the biological context of melanoma. Functional assays revealed positive effects of XIST on cellular functions (proliferation, migration, and invasion) that diminished after upregulation of miR-139-5p. These results suggest that XIST is a cancer-promoting gene in melanoma and acts via miR-139-5p. Our results are consistent with those of previous studies. Hu et al found that XIST facilitated bladder cancer cellular functions (growth and metastasis) by sponging miR-139-5p, ${ }^{23}$ and Mo et al similarly found that XIST facilitated hepatocellular carcinoma cell growth by sponging miR-139-5p. ${ }^{24}$

We further explored the potential target mRNA regulated by miR-139-5p. TargetScan and StarBase 3.0 software, dualluciferase reporter system and Western blot assay were utilized to identify Rho-associated coiled-coil containing 


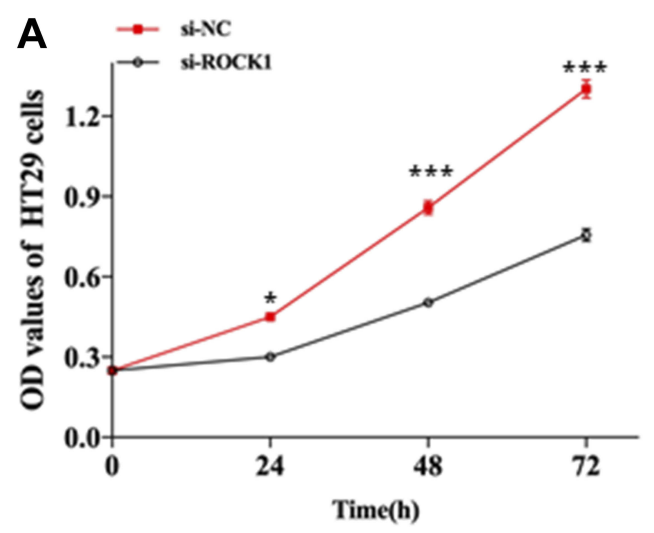

B

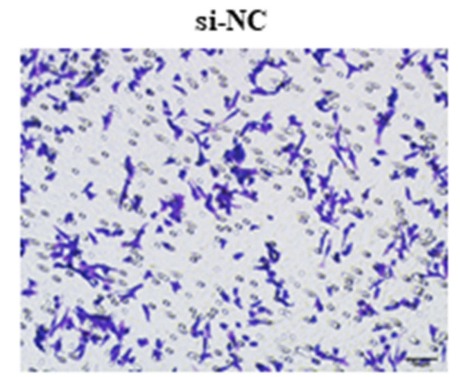

C

$\mathrm{Oh}$
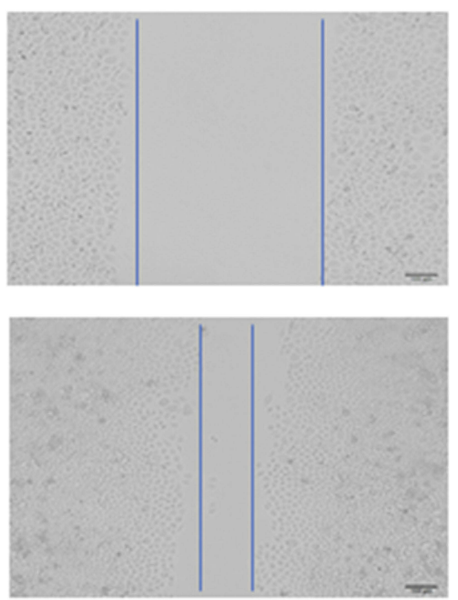

si-NC

$24 \mathrm{~h}$

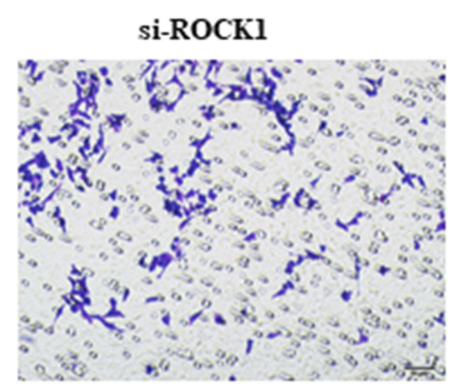

si-ROCK1
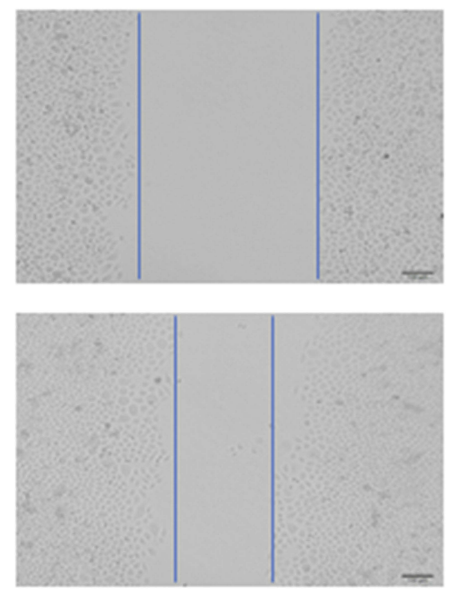

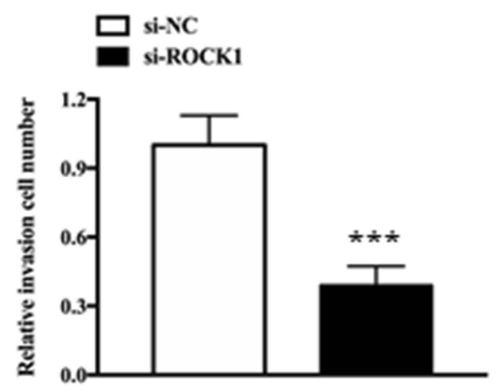

Figure 5 The role of ROCKI in cellular function.

Notes: (A) Cell proliferation in HS-I cells after transfection with si-ROCKI or si-NC. (B) Cell invasion in HS-I cells after transfection with si-ROCKI or Si-NC. (C) Cell migration in HS-I cells after transfection with si-ROCKI or si-NC. All bars are $100 \mu \mathrm{m}$. $* \mathrm{P}<0.05, * * * \mathrm{P}<0.001$.

Abbreviations: OD, optical density; si-NC, scrambled RNA for negative control.

protein kinase 1 (ROCK1) as a direct target of miR-139-5p. ROCK1 was reported to interact with miRNA and lncRNA, regulating cellular migration, invasion, and metastasis during cancer pathogenesis. For example, miR-202-5p suppress tumor cell migration and invasion in osteosarcoma, with these effects weakened by upregulation of ROCK $1 .{ }^{25}$ Moreover, knockdown of ROCK1 inhibited the positive effects of the lncRNA ITGB1 on hepatoma carcinoma cell growth, migration, and invasion. ${ }^{26}$ In osteosarcoma, upregulation of lncRNA EPEL has been demonstrated to facilitate tumor cell migration and invasion by inducing upregulation of ROCK $1 .{ }^{27}$ In osteosarcoma, ROCK1 participates in the regulation of SNHG5 in tumor growth, invasion, and migration. ${ }^{28}$ In this research, the lncRNA XIST was found to sponge miR-139-5p, thereby reducing ROCK1 levels.

In conclusion, the IncRNA XIST is downregulated in melanoma and facilitates tumor cell proliferation, migration, and invasion via the miR-139-5p/ROCK1 pathway. These findings demonstrate the potential of XIST as a future target for melanoma therapy. 

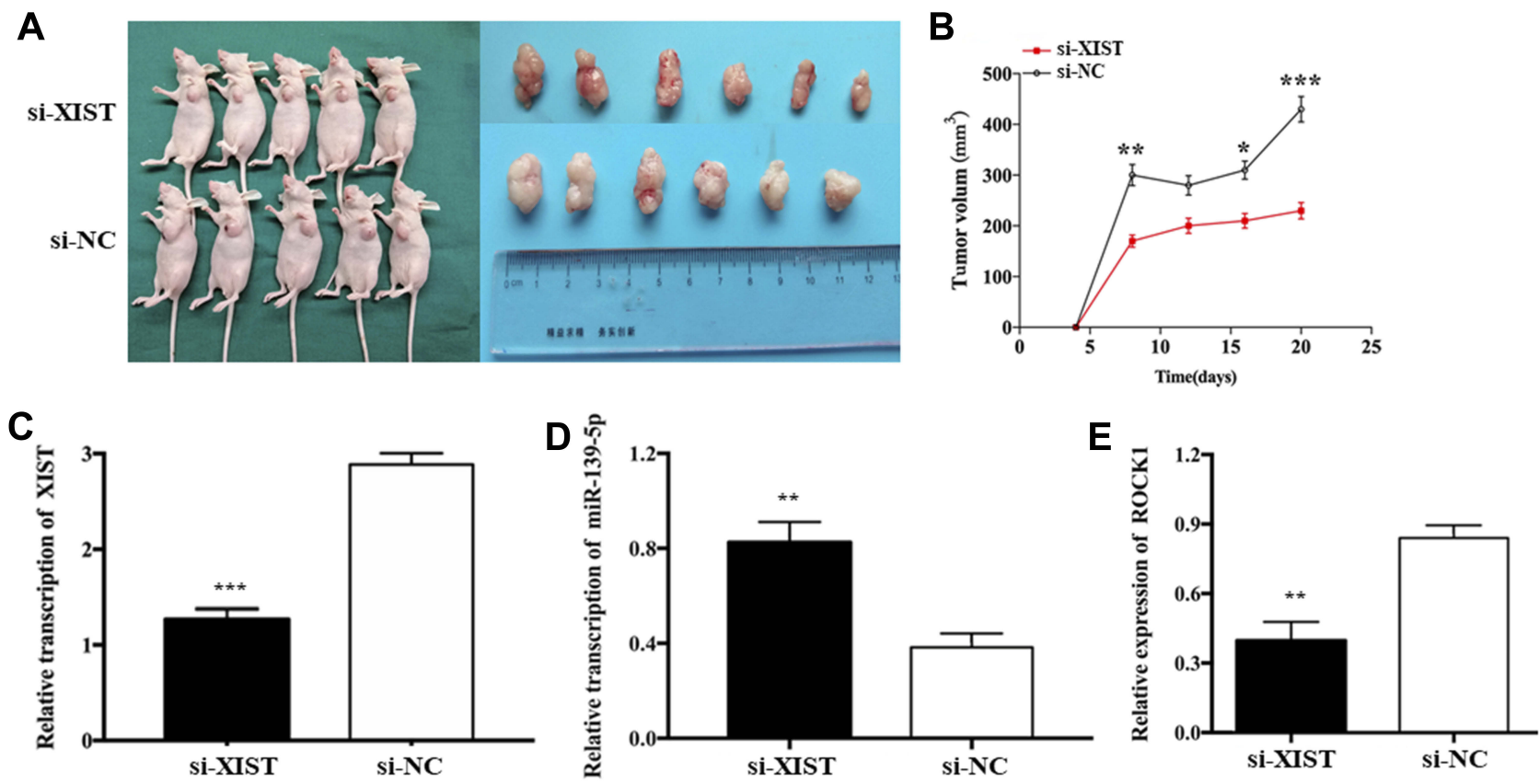

Figure 6 XIST promoted tumor growth of TNBC cells in vivo.

Notes: (A and B) Tumors were obtained from nude mice injected subcutaneously with HS-I cells transfected with si-XIST and si-NC and tumor weights were measured, respectively. (C-E) qRT-PCR analysis of XIST, miR-I39-5p, and ROCKI expressions in the tumor growing from transplanted HS-I cells with si-XIST and si-NC. *P<0.05, $* * \mathrm{P}<0.01, * * * \mathrm{P}<0.001$.

\section{Disclosure}

The authors report no conflicts of interest in this work.

\section{References}

1. Ferlay J, Soerjomataram I, Dikshit R, et al. Cancer incidence and mortality worldwide: sources, methods and major patterns in GLOBOCAN 2012. Int J Cancer. 2015;136(5):E359-E386. doi:10.1002/ijc.29210

2. Yang S, Xu J, Zeng X. A six-long non-coding RNA signature predicts prognosis in melanoma patients. Int J Oncol. 2018;52(4):1178-1188. doi:10.3892/ijo.2018.4268

3. Gao J, Zeng K, Liu Y, Gao L, Liu L. LncRNA SNHG5 promotes growth and invasion in melanoma by regulating the miR-26a-5p/ TRPC3 pathway. Onco Targets Ther. 2019;12:169-179. doi:10.21 47/OTT.S184078

4. Hosono Y, Niknafs YS, Prensner JR, et al. Oncogenic role of THOR, a conserved cancer/testis long non-coding RNA. Cell. 2017;171 (7):1559-1572.e1520. doi:10.1016/j.cell.2017.11.040

5. Fang Y, Fullwood MJ. Roles, functions, and mechanisms of long non-coding RNAs in cancer. Genomics Proteomics Bioinformatics. 2016;14(1):42-54. doi:10.1016/j.gpb.2015.09.006

6. Yang Y, Zhang J, Chen X, et al. LncRNA FTX sponges miR-215 and inhibits phosphorylation of vimentin for promoting colorectal cancer progression. Gene Ther. 2018;25(5):321-330. doi:10.1038/s41434-0180026-7

7. Xing F, Liu Y, Wu SY, et al. Loss of XIST in breast cancer activates MSN-c-Met and reprograms microglia via exosomal miRNA to promote brain metastasis. Cancer Res. 2018;78(15):4316-4330. doi:10.1158/0008-5472.CAN-18-1102

8. Liu A, Liu L, Lu H. LncRNA XIST facilitates proliferation and epithelial-mesenchymal transition of colorectal cancer cells through targeting miR-486-5p and promoting neuropilin-2. J Cell Physiol. 2019;234(8):13747-13761.
9. Hu B, Shi G, Li Q, Li W, Zhou H. Long noncoding RNA XIST participates in bladder cancer by downregulating p53 via binding to TET1. J Cell Biochem. 2019;120(4):6330-6338. doi:10.1002/jcb.v120.4

10. Wang L, Hu K, Chao Y. MicroRNA-1301 inhibits migration and invasion of osteosarcoma cells by targeting BCL9. Gene. 2018;679:100-107. doi:10.1016/j.gene.2018.08.078

11. Zhu H, Zheng T, Yu J, Zhou L, Wang L. LncRNA XIST accelerates cervical cancer progression via upregulating Fus through competitively binding with miR-200a. Biomed Pharmacother. 2018;105:789-797. doi:10.1016/j.biopha.2018.05.053

12. Zheng R, Lin S, Guan L, et al. Long non-coding RNA XIST inhibited breast cancer cell growth, migration, and invasion via miR-155/ CDX1 axis. Biochem Biophys Res Commun. 2018;498(4): 1002-1008. doi:10.1016/j.bbrc.2018.03.104

13. Du Y, Weng XD, Wang L, et al. LncRNA XIST acts as a tumor suppressor in prostate cancer through sponging miR-23a to modulate RKIP expression. Oncotarget. 2017;8(55):94358-94370.

14. Wang L, Feng Z, Wang X, Wang X, Zhang X. DEGseq: an R package for identifying differentially expressed genes from RNA-seq data. Bioinformatics. 2010;26(1):136-138. doi:10.1093/bioinformatics/ btp612

15. Rivetti di Val Cervo P, Lena AM, Nicoloso M, et al. p63-microRNA feedback in keratinocyte senescence. Proc Natl Acad Sci U S A. 2012;109(4):1133-1138. doi:10.1073/pnas.1112257109

16. Vasaikar SV, Straub P, Wang J, Zhang B. LinkedOmics: analyzing multi-omics data within and across 32 cancer types. Nucleic Acids Res. 2017;46(D1):D956-D963. doi:10.1093/nar/gkx1090

17. Pan B, Lin X, Zhang L, Hong W, Zhang Y. Long noncoding RNA $\mathrm{X}$-inactive specific transcript promotes malignant melanoma progression and oxaliplatin resistance. Melanoma Res. 2019;29(3):254-262. doi:10.1097/CMR.0000000000000560

18. Chen X, Xiong D, Ye L, et al. Up-regulated lncRNA XIST contributes to progression of cervical cancer via regulating miR-140-5p and ORC1. Cancer Cell Int. 2019;19:45. doi:10.1186/s12935-0190744-y 
19. Ma L, Zhou Y, Luo X, Gao H, Deng X, Jiang Y. Long non-coding RNA XIST promotes cell growth and invasion through regulating miR-497/MACC1 axis in gastric cancer. Oncotarget. 2017;8 (3):4125-4135. doi:10.18632/oncotarget. 13670

20. Paraskevopoulou MD, Hatzigeorgiou AG. Analyzing miRNA-lncRNA interactions. Methods Mol Biol. 2016;1402:271-286.

21. Yang B, Zhang W, Sun D, et al. Downregulation of miR-139-5p promotes prostate cancer progression through regulation of SOX5. Biomed Pharmacother. 2019;109:2128-2135. doi:10.1016/j.biopha.2018.09.029

22. Shi YK, Guo YH. MiR-139-5p suppresses osteosarcoma cell growth and invasion through regulating DNMT1. Biochem Biophys Res Commun. 2018;503(2):459-466. doi:10.1016/j.bbrc.2018.04.124

23. Hu Y, Deng C, Zhang H, Zhang J, Peng B, Hu C. Long non-coding RNA XIST promotes cell growth and metastasis through regulating miR-139$5 \mathrm{p}$ mediated Wnt/beta-catenin signaling pathway in bladder cancer. Oncotarget. 2017;8(55):94554-94568. doi:10.18632/oncotarget.21791

24. Mo Y, Lu Y, Wang P, et al. Long non-coding RNA XIST promotes cell growth by regulating miR-139-5p/PDK1/AKT axis in hepatocellular carcinoma. Tumour biology : the journal of the International Society for Oncodevelopmental Biology and Medicine. Feb 2017;39 (2):1010428317690999
25. Li C, Ma D, Yang J, Lin X, Chen B. miR-202-5p inhibits the migration and invasion of osteosarcoma cells by targeting ROCK1. Oncol Lett. 2018;16(1):829-834. doi:10.3892/ol.2018. 8694

26. Huang L, Li X, Gao W. Long non-coding RNA linc-ITGB1 promotes cell proliferation, migration, and invasion in human hepatoma carcinoma by up-regulating ROCK1. Biosci Rep. 2018;38(5). doi:10.1042/ BSR20181289

27. Chen S, Liu Z, Lu S, Hu B. EPEL promotes the migration and invasion of osteosarcoma cells by upregulating ROCK1. Oncol Lett. 2019;17(3):3133-3140. doi:10.3892/ol.2019.9975

28. Wang Z, Wang Z, Liu J, Yang H. Long non-coding RNA SNHG5 sponges miR-26a to promote the tumorigenesis of osteosarcoma by targeting ROCK1. Biomed Pharmacother. 2018;107:598-605. doi:10.1016/j.biopha.2018.08.025

\section{Publish your work in this journal}

OncoTargets and Therapy is an international, peer-reviewed, open access journal focusing on the pathological basis of all cancers, potential targets for therapy and treatment protocols employed to improve the management of cancer patients. The journal also focuses on the impact of management programs and new therapeutic agents and protocols on patient perspectives such as quality of life, adherence and satisfaction. The manuscript management system is completely online and includes a very quick and fair peer-review system, which is all easy to use. Visit http://www.dovepress.com/ testimonials.php to read real quotes from published authors. 